\title{
The Spin-orbit resonance of Mercury: a Hamiltonian approach
}

\author{
S. D'Hoedt and A. Lemaitre \\ Department of Mathematics, FUNDP, Namur, B-5000, Belgium \\ emails: sandrine.dhoedt@fundp.ac.be, anne.lemaitre@fundp.ac.be
}

\begin{abstract}
One of the main characteristics of Mercury is its 3:2 spin-orbit resonance, combined with a 1:1 resonance between the orbital node of its orbit and the angle describing the precession of the rotation axis, both measured on the ecliptic plane. We build an analytical model, using Hamiltonian formalism, that takes into account this phenomenon thanks to the introduction of three resonant variables and conjugated momenta. We calculate the equilibria corresponding to four different configurations, which means four completely different values of the (ecliptic) obliquity; in particular, we focus on the present (stable) situation of Mercury, and thanks to several canonical transformations, we obtain, near the equilibrium, three pairs of angle-action variables, and consequently, three basic frequencies. Let us note that the model is as simple as possible: the gravitational potential is limited to the second degree terms (the only ones for which a value can be presently given), and the orbit of Mercury is Keplerian. The numerical values obtained by our simplified model are validated by the coherence with existing complete numerical models.
\end{abstract}

\section{Introduction}

Motivated by the projects of space missions like BepiColombo $†$ or MESSENGER $\ddagger$, we have in mind to build an analytical resonant spin-orbit model for the present situation of Mercury. We use a very classical Hamiltonian formalism, starting with a kernel model, and adding step by step the different perturbations.

In this paper, we first present the kernel, in the form of a three-degrees-of-freedom Hamiltonian system, averaged over the short periods; it succeeds in describing the libration about the resonant spin-orbit 3:2 motion by three pairs of action-angle variables, obtained after a succession of simplifications and canonical changes of variables. The three basic frequencies of the system are in complete agreement with the very recent values obtained numerically by Rambaux \& Bois (2003).

The basic hypotheses are the following: Mercury is a rigid non-spherical body, the gravity field is truncated after the second degree terms (the only ones for which we have significant numerical values), the orbital motion of Mercury is Keplerian. Let us remark that the obliquity is not put to zero and in the last part, the spin axis is not parallel to the third axis of inertia.

All the computations are performed with the software Mathematica with the parameters values taken in Anderson et al. (1987) and ESA-SCI (2000).

$\dagger$ Mission of the European Space Agency and ISAS, Japan's Institute of Space Astronautical Sciences

$\ddagger$ MErcury Surface Space ENvironment, Geochemistry and Ranging, space mission of NASA 


\section{Coordinate Choices}

We are going to work with four reference frames centered at Mercury's center of mass:

- $\left(X_{0}, Y_{0}, Z_{0}\right)$ inertial frame with $X_{0}$ and $Y_{0}$ in the ecliptic plane (fixed at some epoch),

- $\left(X_{1}, Y_{1}, Z_{1}\right)$ orbital frame with $Z_{1}$ perpendicular to the orbit plane,

- $\left(X_{2}, Y_{2}, Z_{2}\right)$ with $Z_{2}$ pointing to the spin axis direction and $X_{2}$ directed along the ascending node of the equatorial plane on the ecliptic plane and

- $\left(X_{3}, Y_{3}, Z_{3}\right)$ with $Z_{3}$ in the direction of the axis of greatest inertia and $X_{3}$ in the direction of the axis of smallest inertia.

The choice of the ecliptic frame as the inertial frame is motivated by our intention to introduce further planetary perturbations on the model.

These frames are linked together (see Fig. 1$)$ by three sets of Euler's angles $\left(h, K,_{-}\right)$, $(g, J, l)$ and $\left(\Omega_{o}, i_{o}, \omega_{o}\right)$ (where the subscript $o$ stands for "orbital") with $\Omega_{o}$ the ascending node longitude, $i_{o}$ the inclination and $\omega_{o}$ the pericenter argument.
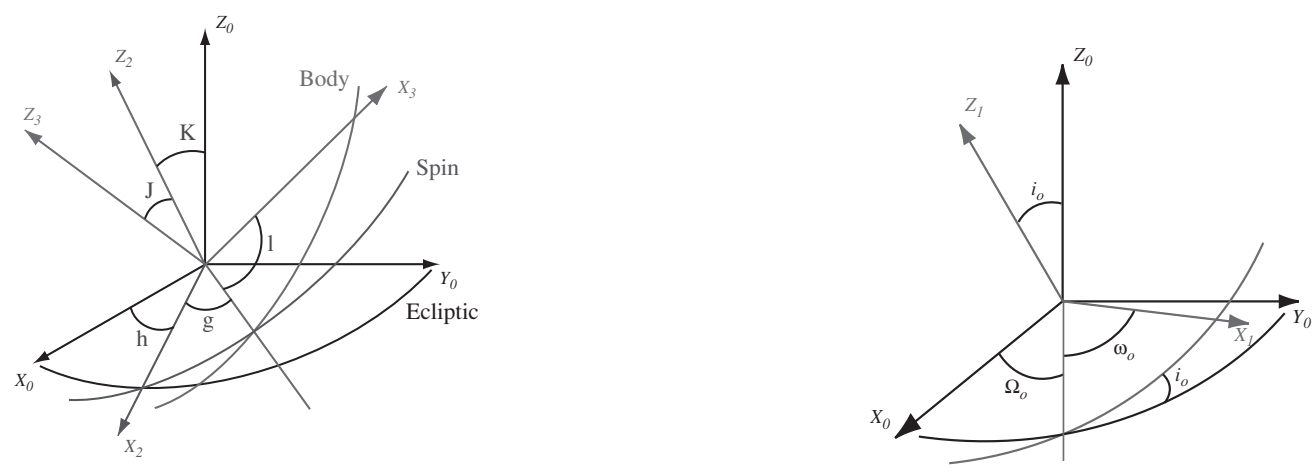

Figure 1. On the left side, the $\left(X_{0}, Y_{0}, Z_{0}\right),\left(X_{2}, Y_{2}, Z_{2}\right)$ and $\left(X_{3}, Y_{3}, Z_{3}\right)$ frames with $\left(h, K,_{-}\right)$ being Euler's angles related to $\left(X_{0}, Y_{0}, Z_{0}\right)$ and $\left(X_{2}, Y_{2}, Z_{2}\right)$ and $(g, J, l)$ being Euler's angles related to $\left(X_{2}, Y_{2}, Z_{2}\right)$ and $\left(X_{3}, Y_{3}, Z_{3}\right)$. On the right side, the $\left(X_{1}, Y_{1}, Z_{1}\right)$ frame with $\left(\Omega_{o}, i_{o}, \omega_{o}\right)$ being Euler's angles related to $\left(X_{0}, Y_{0}, Z_{0}\right)$ and $\left(X_{1}, Y_{1}, Z_{1}\right)$. All of them are centered at Mercury's center of mass.

In fact, to describe the motion of Mercury, we need two sets of canonical variables: the orbital ones, and the rotational ones.

A first classical orbital choice would be to take Delaunay's elements, related to the ecliptic plane (Mercury being a mass reduced to its center of mass):

$$
L_{o}, G_{o}, H_{o}, l_{o}, g_{o}, h_{o}
$$

The capital letters designate the conjugated momenta associated to the angles $l_{o}$ (mean anomaly), $g_{o}=\omega_{o}$ and $h_{o}=\Omega_{o}$, and are classically defined as

$$
L_{o}=m \sqrt{\mu a}, \quad G_{o}=L_{o} \sqrt{1-e^{2}}, \quad H_{o}=G_{o} \cos i_{o},
$$

with $e$ the eccentricity and $a$ the semi-major axis of Mercury's orbit. $\mu$ is $\mathrm{G}(m+M)$ in first approximation, $\mathrm{G}$ is the universal constant of gravitation, $m$ is the mass of Mercury and $M$ this of the Sun. 
For the rotation, following Deprit (1967) and Kinoshita (1972), we adopt Andoyer's variables

$$
L, G, H, l, g, h,
$$

$G$ being the norm of the angular momentum, $L=G \cos J$ the projection of the angular momentum on $Z_{3}$ and $H=G \cos K$ the projection of the angular momentum on $Z_{0}$.

The problem of these Andoyer's variables is that angular variables are not well-defined if $K$ or/and $J$ are zero but their sum is always well-defined. This is why we have chosen a new set of partially non singular variables $\left(\Lambda_{1}, \Lambda_{2}, \Lambda_{3}, \lambda_{1}, \lambda_{2}, \lambda_{3}\right)$ such that the angular variables are:

$$
\lambda_{1}=l+g+h, \quad \lambda_{2}=-l, \quad \lambda_{3}=-h
$$

and their conjugated momenta:

$$
\Lambda_{1}=G, \Lambda_{2}=G-L=G(1-\cos J), \Lambda_{3}=G-H=G(1-\cos K) .
$$

Let us remark that the transformation from Andoyer's variables to the new one is canonical.

\section{Model of rotation}

Let us introduce our main hypothesis for this first approach: Mercury is assumed to move on a fixed elliptic orbit, with the present orbital parameters: $a_{o}=57.9 \times 10^{6} \mathrm{~km}$, $e_{o}=0.206, i_{o}=7^{\circ}$. Without any planetary perturbation, the Hamiltonian can be written:

$$
\mathcal{H}=-\frac{m^{3} \mu^{2}}{2 L_{o}^{2}}+T\left(\Lambda_{1}, \Lambda_{2}, \Lambda_{3}, \lambda_{1}, \lambda_{2}, \lambda_{3}\right)+V_{G}\left(\Lambda_{1}, \Lambda_{2}, \Lambda_{3}, \lambda_{1}, \lambda_{2}, \lambda_{3}\right)
$$

where $T$ is the kinetic energy and $V_{G}$ the gravity potential. One can show (Deprit 1967) that

$$
T=\frac{\left(\Lambda_{1}-\Lambda_{2}\right)^{2}}{2 I_{3}}+\frac{1}{2}\left(\Lambda_{1}^{2}-\left(\Lambda_{1}-\Lambda_{2}\right)^{2}\right)\left(\frac{\sin ^{2} \lambda_{2}}{I_{1}}+\frac{\cos ^{2} \lambda_{2}}{I_{2}}\right)
$$

where $I_{1}, I_{2}, I_{3}$ are the principal inertia momenta with $I_{1}<I_{2}<I_{3}$.

As Peale (1974) and other authors did, and because only few data about Mercury are known, we will limit the development of $V_{G}$ to the second order in spherical harmonics, the other ones will be considered as perturbations on the basic model:

$$
V_{G}=-\frac{G M m}{r}\left(\frac{R_{e}}{r}\right)^{2}\left[C_{2}^{0} P_{2}(\sin \theta)+C_{2}^{2} P_{2}^{2}(\sin \theta) \cos 2 \varphi\right]
$$

where $P_{2}$ and $P_{2}^{2}$ refer to the second order Legendre's polynomial and associated polynomial, $R_{e}$ is Mercury's equatorial radius, $r$ the distance between the Sun and Mercury centers of mass, $\theta$ and $\varphi$ the latitude and longitude of a Mercury's surface element, in the frame $\left(X_{3}, Y_{3}, Z_{3}\right)$.

Let us now express $V_{G}$ in terms of Cartesian coordinates:

$$
\left\{\begin{array}{l}
\bar{x}_{3}=\cos \varphi \cos \theta \\
\bar{y}_{3}=\sin \varphi \cos \theta \\
\bar{z}_{3}=\sin \theta
\end{array}\right.
$$

where $\left(\bar{x}_{3}, \bar{y}_{3}, \bar{z}_{3}\right)$ is the unit vector in the direction of the perturbing body (the subscript "3" being used to recall that we are working in the frame $\left.\left(X_{3}, Y_{3}, Z_{3}\right)\right)$. Thus replacing 
the Legendre polynomials by their expressions, we find:

$$
V_{G}=-\frac{G M m}{r^{3}}\left(R_{e}\right)^{2}\left[\frac{C_{2}^{0}}{2}\left(2 \bar{z}_{3}^{2}-\bar{x}_{3}^{2}-\bar{y}_{3}^{2}\right)+3 C_{2}^{2}\left(\bar{x}_{3}^{2}-\bar{y}_{3}^{2}\right)\right]
$$

However, the purpose being to write the potential in our canonical set of rotational $\left(\Lambda_{1}, \Lambda_{2}, \Lambda_{3}, \lambda_{1}, \lambda_{2}, \lambda_{3}\right)$ and orbital $\left(L_{o}, G_{o}, H_{o}, l_{o}, g_{o}, h_{o}\right)$ variables, let us express the vector $\left(\bar{x}_{3}, \bar{y}_{3}, \bar{z}_{3}\right)$ in the orbital frame:

$$
\begin{array}{r}
\left(\begin{array}{c}
\bar{x}_{3} \\
\bar{y}_{3} \\
\bar{z}_{3}
\end{array}\right)=R_{3}\left(-\lambda_{2}\right) R_{1}(J) R_{3}\left(\lambda_{1}+\lambda_{2}+\lambda_{3}\right) R_{1}(K) R_{3}\left(-\lambda_{3}\right) \times \\
R_{3}\left(-h_{o}\right) R_{1}\left(-i_{o}\right) R_{3}\left(-g_{o}\right)\left(\begin{array}{c}
\cos v_{o} \\
\sin v_{o} \\
0
\end{array}\right)
\end{array}
$$

where $v_{o}$ is the true anomaly, $R_{i}$ are rotation matrices (their subscript is the number of the rotation axis).

\section{Kernel model: 2 degrees of freedom}

For this kernel model, we are going to take $J=0$, which means that the spin axis $Z_{2}$ and the third principal axis of inertia $Z_{3}$ coincide. The coordinates change becomes:

$$
\begin{aligned}
\left(\begin{array}{c}
\bar{x}_{3} \\
\bar{y}_{3} \\
\bar{z}_{3}
\end{array}\right)=R_{3}\left(\lambda_{1}+\lambda_{3}\right) R_{1}(K) R_{3}\left(-\lambda_{3}\right) \times \\
\qquad R_{3}\left(-h_{o}\right) R_{1}\left(-i_{o}\right) R_{3}\left(-g_{o}\right)\left(\begin{array}{c}
\cos v_{o} \\
\sin v_{o} \\
0
\end{array}\right)
\end{aligned}
$$

Thus replacing (4.1) in (3.1) and using the relation $L_{o}=m \sqrt{\mu a}$ and the well known developments in eccentricity, up to the order 3 of Brouwer \& Clemence (1961) allow us to introduce missing orbital variables $l_{o}$ and $L_{o}$ in the potential expression $V_{G}$ :

$$
\begin{aligned}
V_{G}= & -\frac{G M m^{7}}{L_{o}^{6}} \mu^{3}\left(R_{e}\right)^{2} \times \\
& \left\{\frac { 1 } { 2 } C _ { 2 } ^ { 0 } \left[a_{000}+\sum_{i=1}^{5}\left(a_{00 i} \cos \left(i l_{o}\right)+b_{00 i} \sin \left(i l_{o}\right)\right)\right.\right. \\
& \left.+\sum_{j=1}^{2} \sum_{i=-5}^{5}\left(a_{0 j i} \cos \left(j \lambda_{3}+i l_{o}\right)+b_{0 j i} \sin \left(j \lambda_{3}+i l_{o}\right)\right)\right] \\
+ & \left.3 C_{2}^{2}\left[\sum_{j=0}^{4} \sum_{i=-5}^{5}\left(a_{2 j i} \cos \left(2 \lambda_{1}+j \lambda_{3}+i l_{o}\right)+b_{2 j i} \sin \left(2 \lambda_{1}+j \lambda_{3}+i l_{o}\right)\right)\right]\right\}
\end{aligned}
$$

where $a$ and $b$ depend on $K, h_{o}, i_{o}, g_{o}$ and $e$, with $h_{o}, i_{o}, g_{o}$ and $e$ considered as constants. So, in our basic model, $V_{G}$ is only function of 3 momenta and 3 angles: $\Lambda_{1}$ and $\Lambda_{3}$ (through $K), L_{o}, \lambda_{1}, \lambda_{3}$ and $l_{o}$. 


\section{The main resonant angle}

Let us recall again that Mercury is in a 3:2 spin-orbit resonance which can be expressed in our variables by:

$$
\dot{\lambda}_{1}=\frac{3}{2} \dot{l}_{o} .
$$

The two sets of canonical variables (orbital and rotational) are then mixed in this commensurability.

Let us thus take

$$
\sigma=\frac{2 \lambda_{1}-3 l_{o}}{2}
$$

as resonant angle and $\Lambda_{1}$ as its conjugated momentum. In order to keep a canonical transformation, we must associate to $l_{o}$ a new conjugated momentum $\Lambda_{o}=L_{o}+\frac{3}{2} \Lambda_{1}$.

If we replace by these new variables in (4.2) and after averaging on $l_{o}$, only a few terms stay ( $<>$ stands for "averaged"):

$$
\begin{aligned}
<V_{G}>= & -\frac{G M m^{7}}{\left(\Lambda_{0}-\frac{3}{2} \Lambda_{1}\right)^{6}} \mu^{3}\left(R_{e}\right)^{2} \\
& \times\left(\frac{1}{2} C_{2}^{0}\left(a_{000}+\sum_{k=1}^{2}\left(a_{0 k 0} \cos \left(k \lambda_{3}\right)+b_{0 k 0} \sin \left(k \lambda_{3}\right)\right)\right)\right. \\
& \left.+3 C_{2}^{2}\left(\sum_{k=0}^{4}\left(a_{2 k 0} \cos \left(2 \sigma+k \lambda_{3}\right)+b_{2 k 0} \sin \left(2 \sigma+k \lambda_{3}\right)\right)\right)\right)
\end{aligned}
$$

\section{Simplified Hamiltonian}

Considering that the eccentricity $e \sim 0.206$, we are going to keep the terms in $\left(1+\frac{3 e^{2}}{2}\right)$ and $\left(\frac{7 e}{2}-\frac{123 e^{3}}{16}\right)$ but not in $e^{3}$ only; those terms will act as perturbations on our first model. And so, we obtain a two degrees of freedom Hamiltonian (see D'Hoedt \& Lemaitre (2004) for details):

$$
\begin{aligned}
<\mathcal{H}>= & \frac{\Lambda_{1}^{2}}{2 I_{3}}-\frac{m^{3} \mu^{2}}{2\left(\Lambda_{0}-\frac{3 \Lambda_{1}}{2}\right)^{2}}-\frac{G M m^{7} \mu^{3} R_{e}^{2}}{\left(\Lambda_{0}-\frac{3 \Lambda_{1}}{2}\right)^{6}} \times \\
& {\left[\frac{1}{2} C_{2}^{0}\left(1+\frac{3 e^{2}}{2}\right)\left(\sum_{i=0}^{2} a_{0 i} \cos \left(i \sigma_{3}\right)\right)\right.} \\
& \left.+3 C_{2}^{2}\left(\frac{7 e}{2}-\frac{123 e^{3}}{16}\right)\left(\sum_{i=0}^{4} a_{2 i} \cos \left(2 \sigma_{1}+i \sigma_{3}\right)\right)\right]
\end{aligned}
$$

with $\sigma_{1}=\sigma-h_{o}-g_{o}, \sigma_{3}=\lambda_{3}+h_{o}$ and where $a_{0 i}$ and $a_{2 i}$ depend on $i_{o}$ and $K$. (The canonical transformation from old to new variables allows us to keep $\Lambda_{1}$ and $\Lambda_{3}$ as conjugated momenta to $\sigma_{1}$ and $\sigma_{3}$.)

After computation of the motion equations, we find that the equilibria for the couple $\left(\sigma_{1}, \sigma_{3}\right)$ are: $(0,0),(\pi / 2,0),(0, \pi),(\pi / 2, \pi)$. Let us notice that the last two geometrical configurations are the same as the first two ones. 
For each couple $\left(\sigma_{1}, \sigma_{3}\right)$ we find the different values of the (ecliptic) obliquity $K$ :

if $\left(\sigma_{1}, \sigma_{3}\right)=(0,0)$, it leads to four values of the ecliptic obliquity (two of them are dependent on the numerical values of the coefficients $C_{0}^{2}$ and $\left.C_{2}^{2}\right)$ :

$$
\begin{aligned}
& \sin \left(i_{0}-K\right)=0 \quad \text { or } \\
& \cos \left(i_{0}-K\right)=\frac{C_{2}^{2}\left(\frac{7 e}{2}-\frac{123 e^{3}}{16}\right)}{C_{0}^{2}\left(1+\frac{3 e^{2}}{2}\right)-C_{2}^{2}\left(\frac{7 e}{2}-\frac{123 e^{3}}{16}\right)}
\end{aligned}
$$

if $\left(\sigma_{1}, \sigma_{3}\right)=\left(\frac{\pi}{2}, 0\right)$, it also leads to four values of the ecliptic obliquity and we can see that the first two are the same $\left(K=i_{o}\right.$ and $\left.K=\pi+i_{o}\right)$ :

$$
\begin{aligned}
& \sin \left(i_{0}-K\right)=0 \quad \text { or } \\
& \cos \left(i_{0}-K\right)=\frac{-C_{2}^{2}\left(\frac{7 e}{2}-\frac{123 e^{3}}{16}\right)}{C_{0}^{2}\left(1+\frac{3 e^{2}}{2}\right)+C_{2}^{2}\left(\frac{7 e}{2}-\frac{123 e^{3}}{16}\right)}
\end{aligned}
$$

Finally for each $K$, we can find numerically the value of $\Lambda_{1}$ (see Table 1 ) and a fortiori the value of $\Lambda_{3}$.

The numerical values shown in the table below are calculated with $R_{e}$, the equatorial radius of Mercury, $M$, the mass of Mercury, and the year chosen respectively as unities of length, mass and time.

\begin{tabular}{|c|r|r|r|}
\hline$\left(\sigma_{1}, \sigma_{3}\right)$ & $\left(i_{o}-K\right)(\mathrm{deg})$ & $K(\mathrm{deg})$ & $\Lambda_{1}\left(\frac{\mathrm{mRe}^{2}}{\text { year }}\right) \mid$ \\
\hline \multirow{3}{*}{$(0,0)$} & 0 & $K_{1}=7$ & 13.303 \\
\cline { 2 - 4 } & 180 & $K_{2}=180+7$ & 13.303 \\
\cline { 2 - 4 } & -95.332 & $K_{3}=102.332$ & 13.303 \\
\cline { 2 - 4 } & 95.332 & $K_{4}=-88.332$ & 13.303 \\
\hline \multirow{3}{*}{$\left(\frac{\pi}{2}, 0\right)$} & 0 & $K_{1}=7$ & 13.303 \\
\cline { 2 - 4 } & 180 & $K_{2}=180+7$ & 13.303 \\
\cline { 2 - 4 } & -83.446 & $K_{5}=90.446$ & 13.303 \\
\cline { 2 - 4 } & 83.446 & $K_{6}=-76.446$ & 13.303 \\
\hline
\end{tabular}

Table 1. Equilibria in the case $\sigma_{3}=0$.

Let us notice that the values of $\Lambda_{1}$ seem equal, but they differ from each other when we consider more decimals.

We can check that the first equilibrium (the present situation of Mercury) is stable.

\section{Frequency of the two angular variables}

Of course, Mercury is not blocked at the exact 3:2 resonance, but performs a small libration about this equilibrium. To measure the frequency of this libration, we perform a translation to the equilibrium and after successive canonical transformations we can write our Hamiltonian in angle-action coordinates and find the frequencies of $\sigma_{1}$ and $\sigma_{3}$ :

$$
\nu\left(\sigma_{1}\right)=0.396234 \frac{1}{\text { year }}, \quad \nu\left(\sigma_{3}\right)=0.00589928 \frac{1}{\text { year }}
$$

In terms of periods, $\sigma_{1}$ has a period of $15.8573 \mathrm{yr}$ and $\sigma_{3}$ a period of $1065.08 \mathrm{yr}$, which coincides with the results of Rambaux \& Bois (2003). 


\section{Introduction of the third degree of freedom}

Let us now take into account that $J \neq 0, \lambda_{2}$ is thus now well defined. The potential $<V_{G}>$ becomes:

$$
\begin{aligned}
<V_{G}>= & -\frac{G M m^{7}}{\left(\Lambda_{0}-\frac{3}{2} \Lambda_{1}\right)^{6}} \mu^{3}\left(R_{e}\right)^{2} \\
\times & \left(\frac{1}{2} C_{2}^{0}\left(d_{000}+\sum_{k=1}^{2} d_{00 k} \cos \left(k \sigma_{3}\right)+\sum_{k=0}^{4} d_{22 k} \cos \left(2 \sigma_{1}+2 \lambda_{2}+k \sigma_{3}\right)\right)\right. \\
& +3 C_{2}^{2}\left(\sum_{k=-2}^{2} d_{02 k} \cos \left(2 \lambda_{2}+k \sigma_{3}\right)+\sum_{k=0}^{4} d_{20 k} \cos \left(2 \sigma_{1}+k \sigma_{3}\right)\right. \\
& \left.+\sum_{k=0}^{4} d_{24 k} \cos \left(2 \sigma_{1}+4 \lambda_{2}+k \sigma_{3}\right)\right)
\end{aligned}
$$

with $d$ depending on $i_{o}, J, K$ and $e$.

If we express this third degree of freedom in terms of Cartesian coordinates $(\xi, \eta)$ by this canonical transformation

$$
\left\{\begin{array}{l}
\sqrt{2 \Lambda_{2}} \cos \lambda_{2}=\xi \\
\sqrt{2 \Lambda_{2}} \sin \lambda_{2}=\eta,
\end{array}\right.
$$

we can show that $(\xi, \eta)=(0,0)$ is an equilibrium and at this equilibrium we find the same equilibria as previously for the other 2 degrees of freedom.

Once more, Mercury isn't at the exact equilibrium, but performs a small libration about it. Thus, in the same way as previously for $\sigma_{1}$ and $\sigma_{3}$, we find the period of $\lambda_{2}$ which is $584 \mathrm{yr}$.

\section{References}

Anderson, J.D., Colombo, G., Esposito, P.B., Lau, E.L, Trager, G.B. 1987, 'The mass, gravity field and ephemeris of Mercury', Icarus 71, 337-349.

Brouwer, D. \& Clemence, G.M. 1961, 'Methods of celestial mechanics' Academic Press, New York.

Deprit, A. 1967, 'Free Rotation of a Rigid body Studied in the Phase Plane', American Journal of Physics 35 (5), 424-428.

D'Hoedt, S. and Lemaitre, A. 2004, 'The spin-orbit resonant rotation of Mercury: a two degree of freedom Hamiltonian model', Celestial Mechanics, in press.

ESA-SCI 2000, 'BepiColombo, An Interdisciplinary Cornerstone Mission to the Planet Mercury' System and Technology Study Report.

Kinoshita, H. 1972, 'First-Order Perturbations of the Two Finite Body Problem', In Publ. Astron. Soc. Japan 24, 423-457.

Peale, S.J. 1974, 'Possible histories of the obliquity of Mercury' The Astronomical Journal 79, $722-744$.

Rambaux, N. and Bois 2003,'Theory of the Mercury's spin-orbit motion and analysis of its main librations', Astronomy and Astrophysics 413, 381-393 


\section{Discussion}

Jim Message: Are there any observational results on these librations?

SANDRIne D'Hoedt: No, we are expecting two missions. Few things are known about Mercury - very few things.

Myles StAndish: Will a spacecraft get measurements of Mercury's libration?

SANDRINE D'HoEdT: Yes.

Myles StAnDish: ... actual physical measurements, either visual or radar?

SANDRINE D'HoEdT: Yes.

WALTER BRISKEn: There's some evidence that the core of Mercury is rotating differently than the outer shell. Is there any way that this can be incorporated into your model?

SANDRINE D'HoedT: Yes, our Brussels team works on the core of Mercury; we work on the rigid body and then we get the results.

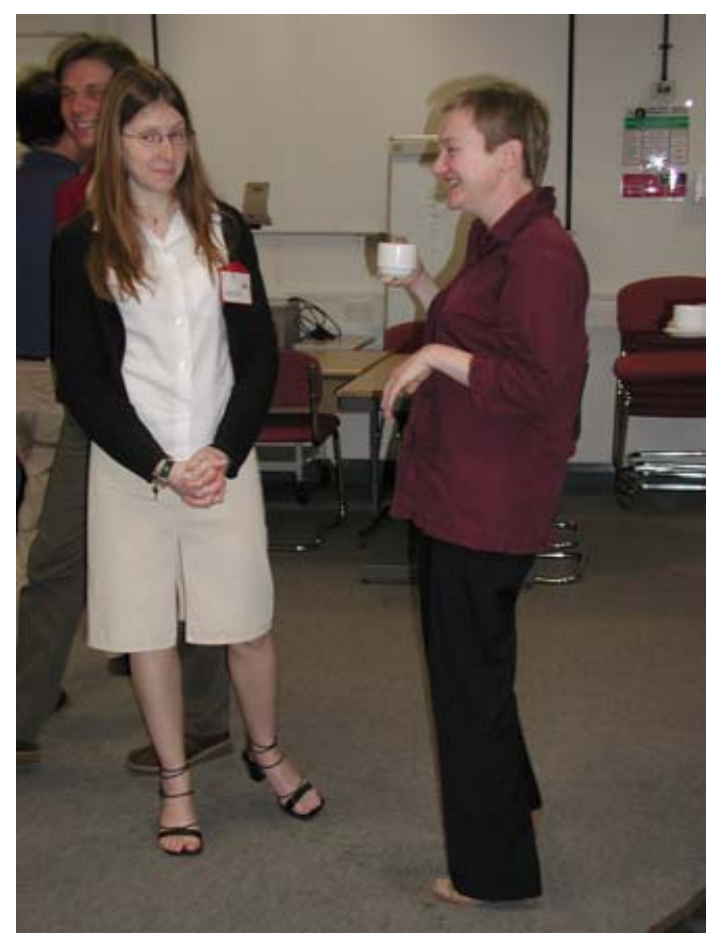

Sandrine D'Hoedt and Anne Lemaitre 